\title{
Effect of Chemical Reaction, Thermo-Diffusion and Dissipation on Unsteady Convective Heat and Mass Transfer in a Vertical Channel with Travelling Thermal Wave
}

\author{
P. Vijaya Lakshmi ${ }^{1}$ and Prof.D.R.V. Prasada Rao \\ ${ }^{1}$ Assistant Professor, Gates Institute of Technology, Gooty,Anantapuramu(D),A.P., India \\ ${ }^{2}$ Professor (Retired), Department of Mathematics, Sri Krishnadevaraya University, \\ Anantapuramu, A.P., India
}

\section{ABSTRACT}

In this paper, we discuss the effect of chemical reaction and Soret effect on unsteady thermal convection flow of a viscous fluid through a porous medium in a vertical channel on whose walls a traveling thermal wave is imposed. The effects of free convective heat and mass transfer flow has been discussed by solving the governing unsteady non-linear equations under perturbation scheme. The velocity, the temperature and the concentration have been analysed for different variations of the governing parameters Sc,So,k,Ec. The rate of heat transfer and the rate of mass transfer have been evaluated numerically for different variations.

Keywords : Chemical Reaction, Thermo-Diffusion, Heat and Mass Transfer, Vertical Channel, Thermal wave.

\section{INTRODUCTION}

Convection fluid flows generated by traveling thermal waves have also received attention due to applications in physical problems. The linearised analysis of these flows has shown that a traveling thermal wave can generate a mean shear flow within a layer of fluid, and the induced mean flow is proportional to the square of the amplitude of the wave. From a physical point of view, the motion induced by traveling thermal waves is quite interesting as a purely fluid-dynamical problem and can be used as a possible explanation for the observed four - day retrograde zonal motion of the upper atmosphere of venus. Also, the heat transfer results will have a definite bearing on the design of oil or gas -fired boilers.

Vajravelu and Debnath [8] have made an interesting and a detailed study of non-linear convection heat transfer and fluid flows, induced by traveling thermal waves. The traveling thermal wave problem was investigated both analytically and experimentally by Whitehead [9] by postulating series expansion in the square of the aspect ratio (assumed small) for both the temperature and flow fields. Whitehead [9] obtained an analytical solution for the mean flow produced by a moving source. Theoretical predictions regarding the ratio of the mean flow velocity to the source speed were found to be good agreement with experimental observations in Mercury which therefore justified the validity of the asymptotic expansion a posteriori. Ravindra [4] has analysed the mixed convection flow of a viscous fluid through a porous medium in a vertical channel. The thermal buoyancy in the flow field is created by a traveling thermal wave imposed on the boundaries. Purushothama Reddy [3] has analysed the unsteady mixed convective effects on the flow induced by imposing traveling thermal waves on the boundaries. Nagaraja [2] has investigated the combined heat and mass transfer effects on the flow of a viscous fluid through a porous medium in a vertical channel, with the traveling thermal waves imposed on the boundaries while the concentration is maintained uniform on the boundaries Sivanjaneya Prasad [5] has analysed heat and mass transfer 
effects on the flow of an incompressible viscous fluid through a porous medium in vertical channel. Sulochana et al [6] have considered the unsteady convective heat and mass transfer through a porous medium due to the imposed traveling thermal wave boundary through a horizontal channel bounded by non -uniform walls. Tanmay Basak et al [7] have analysed the natural convection flows in a square cavity filled with a porous matrix for uniformly and non-uniformly heated bottom wall and adiabatic top wall maintaining crust temperature of cold vertical walls Darcy - Forchheimer model is used to simulate the momentum transfer in the porous medium. Guria and Jana [12] have discussed the two dimensional free and forced convection flow and heat transfer in a vertical wavy channel with traveling thermal waves embedded in a porous medium. The set of non-linear ordinary differential equations are solved analytically. The velocity and temperature fields have been obtained using perturbation

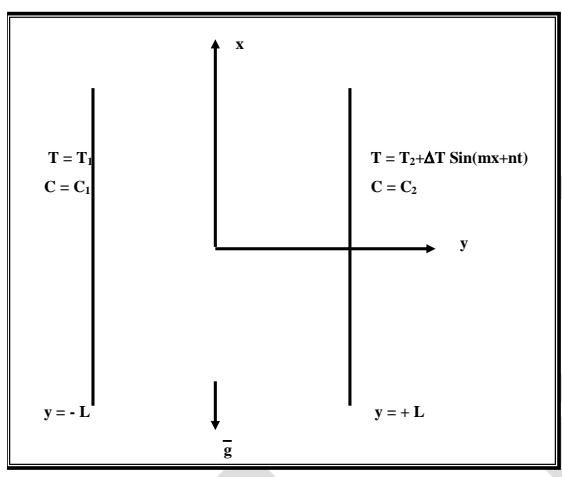

Fig. Configuration of the Problem technique.

\section{FORMULATION OF THE PROBLEM}

We consider the motion of viscous, incompressible fluid through a porous medium in a vertical channel bounded by flat walls. The thermal buoyancy in the flow field is created by a traveling thermal wave imposed on the boundary wall at $\mathrm{y}=\mathrm{L}$ while the boundary at $\mathrm{y}=$ $\mathrm{L}$ is maintained at constant temperature $\mathrm{T}_{1}$. The walls are maintained at constant concentrations. Assuming that the flow takes place at low concentration we neglect the Duffour effect . The Boussinesq approximation is used so that the density variation will be considered only in the buoyancy force. The viscous and Darcy dissipations are taken into account to the transport of heat by conduction and convection in the energy equation. Also the kinematic viscosity $v$, the thermal conducting $\mathrm{k}$ are treated as constants. We choose a rectangular Cartesian system $0(\mathrm{x}, \mathrm{y})$ with $\mathrm{x}$-axis in the vertical direction and $\mathrm{y}$-axis normal to the walls. The walls of the channel are at $y= \pm \mathrm{L}$.

The flow is maintained by a constant volume flux for which a characteristic velocity is defined as

$$
q=\frac{1}{L} \int_{-L}^{L} u d y .
$$

In view of the continuity equation we define the stream function $\psi$ as

$$
\mathrm{u}=-\psi_{\mathrm{y}}, \mathrm{v}=\psi_{\mathrm{x}}
$$

The equations governing the flow in terms of the stream function $\psi$ are

$$
\begin{gathered}
{\left[\left(\nabla^{2} \psi\right)_{t}+\psi_{x}\left(\nabla^{2} \psi\right)_{y}-\psi_{y}\left(\nabla^{2} \psi\right)_{x}\right]=v \nabla^{4} \psi-\beta g\left(T-T_{0}\right)_{y}-\beta^{\bullet} g\left(C-C_{0}\right)_{y}} \\
-\left(\frac{v}{k}\right) \nabla^{2} \psi
\end{gathered}
$$




$$
\begin{gathered}
\rho_{e} C_{p}\left(\frac{\partial T}{\partial t}-\frac{\partial \psi}{\partial y} \frac{\partial T}{\partial x}+\frac{\partial \psi}{\partial x} \frac{\partial T}{\partial y}\right)=\lambda \nabla^{2} T+Q+\mu\left(\left(\frac{\partial^{2} \psi}{\partial y^{2}}\right)^{2}+\left(\frac{\partial^{2} \psi}{\partial x^{2}}\right)^{2}\right)+ \\
\left.+\left(\frac{\mu}{k}\right)\left(\left(\frac{\partial \psi}{\partial x}\right)^{2}+\left(\frac{\partial \psi}{\partial y}\right)^{2}\right)\right) \\
\left(\frac{\partial C}{\partial t}-\frac{\partial \psi}{\partial y} \frac{\partial C}{\partial x}+\frac{\partial \psi}{\partial x} \frac{\partial C}{\partial y}\right)=D_{1} \nabla^{2} C-k_{1} C+k_{11}\left(\frac{\partial^{2} T}{\partial x^{2}}+\frac{\partial^{2} T}{\partial y^{2}}\right)
\end{gathered}
$$

Introducing the non-dimensional variables in (2)- (4) as

$$
x^{\prime}=m x, y^{\prime}=y / L, t^{\prime}=t v m^{2}, \Psi^{\prime}=\Psi / v, \theta=\frac{T-T_{e}}{\Delta T_{e}}, C^{\prime}=\frac{C-C_{1}}{C_{2}-C_{1}}
$$

the governing equations in the non-dimensional form ( after dropping the dashes ) are

$$
\delta R\left(\delta\left(\nabla_{1}^{2} \psi\right)_{t}+\frac{\partial\left(\psi, \nabla_{1}^{2} \psi\right)}{\partial(x, y)}\right)=\nabla_{1}^{4} \psi-\left(\frac{G}{R}\right)\left(\theta_{y}+N C_{y}\right)-D^{-1} \nabla_{1}^{2} \psi
$$

The energy equation in the non-dimensional form is

$$
\begin{gathered}
\delta P\left(\delta \frac{\partial \psi}{\partial t}-\frac{\partial \psi}{\partial y} \frac{\partial \theta}{\partial x}+\frac{\partial \psi}{\partial x} \frac{\partial \theta}{\partial y}\right)=\nabla_{1}^{2} \theta+\alpha+\left(\frac{P R^{2} E_{c}}{G}\right)\left(\left(\frac{\partial^{2} \psi}{\partial y^{2}}\right)^{2}+\delta^{2}\left(\frac{\partial^{2} \psi}{\partial x^{2}}\right)^{2}\right)+ \\
\left.+\left(D^{-1}\right)\left(\delta^{2}\left(\frac{\partial \psi}{\partial x}\right)^{2}+\left(\frac{\partial \psi}{\partial y}\right)^{2}\right)\right)+\frac{4}{3 N_{1}} \frac{\partial^{2} \theta}{\partial y^{2}}
\end{gathered}
$$

The Diffusion equation is

$$
\delta S c\left(\delta \frac{\partial C}{\partial t}-\frac{\partial \psi}{\partial y} \frac{\partial C}{\partial x}+\frac{\partial \psi}{\partial x} \frac{\partial C}{\partial y}\right)=\nabla_{1}^{2} C-k C+\frac{S c S o}{N} \nabla_{1}^{2} \theta
$$

where

$$
R=\frac{q L}{v} \quad \text { (Reynolds number), } G=\frac{\beta g \Delta T_{e} L^{3}}{v^{2}} \quad \text { (Grashof number) } \mathrm{P}=\frac{\mu c_{p}}{k_{1}}(\text { Prandtl number), }
$$

$D^{-1}=\frac{L^{2}}{k}$ (Darcy parameter), $E_{c}=\frac{\beta g L^{3}}{C_{p}}$ (Eckert number), $\delta=m L$ ( Aspect ratio),

$\gamma=\frac{n}{v m^{2}}$ (non-dimensional thermal wave velocity), $S c=\frac{v}{D_{1}}($ Schimdt Number $)$,

$N=\frac{\beta^{*} \Delta C}{\beta \Delta T} \quad$ (Buoyancy ratio) $S_{0}=\frac{k_{11} \Delta T}{v \Delta C} \quad$ (Soret parameter),

$k=\frac{k_{1} L^{2}}{D_{1}} \quad$ (Chemical reaction parameter), $\nabla_{1}^{2}=\delta^{2} \frac{\partial^{2}}{\partial x^{2}}+\frac{\partial^{2}}{\partial y^{2}}$

The boundary conditions in terms of $\psi$ are

$$
\begin{aligned}
& \psi(+1)-\psi(-1)=-1 \\
& \frac{\partial \psi}{\partial x}=0, \frac{\partial \psi}{\partial y}=0 \quad \text { at } y= \pm 1 \\
& \theta(x, y)=1, C(x, y)=0 \quad \text { on } \mathrm{y}=-1 \\
& \theta(x, y)=\operatorname{Sin}(x+y t), \mathrm{C}(\mathrm{x}, \mathrm{y})=1 \quad \text { on } \mathrm{y}=1 \\
& \frac{\partial \theta}{\partial y}=0, \frac{\partial C}{\partial y}=0 \quad \text { at } \quad y=0
\end{aligned}
$$


The value of $\psi$ on the boundary assumes the constant volumetric flow in consistent with the hyphothesis (8). Also the wall temperature varies in the axial direction in accordance with the prescribed arbitrary function $t$.

\section{ANALYSIS OF THE FLOW}

The main aim of the analysis is to discuss the perturbations created over a combined free and forced convection flow due to traveling thermal wave imposed on the boundaries. The perturbation analysis is carried out by assuming that the aspect ratio $\delta$ to be small. We adopt the perturbation scheme and write

$$
\begin{gathered}
\psi(x, y, t)=\psi_{0}(x, y, t)+\delta \psi_{1}(x, y, t)+\delta^{2} \psi_{2}(x, y, t)+ \\
\theta(x, y, t)=\theta_{0}(x, y, t)+\delta \theta_{1}(x, y, t)+\delta^{2} \theta_{2}(x, y, t)+\ldots \ldots \\
C(x, y, t)=C_{0}(x, y, t)+\delta C_{1}(x, y, t)+\delta^{2} C_{2}(x, y, t)+\ldots .
\end{gathered}
$$

On substituting (11) in (6) to (8) and separating the like powers of $\delta$ the equations and respective conditions to the zeroth order are

$$
\begin{aligned}
& \psi_{0, y y y y y}-M_{1}^{2} \psi_{0, y y}=\frac{G}{R}\left(\theta_{0, y}+N C_{O, Y}\right) \\
& \theta_{o, y y}+\alpha+\frac{P E_{c} R^{2}}{G}\left(\psi_{o, y y}\right)^{2}+\frac{P E_{c} M_{1}^{2}}{G}\left(\psi_{o, y}^{2}\right)=0 \\
& C_{O, Y Y}-(k S c) C_{0}=-\frac{S c S o}{N} \theta_{0, y y}
\end{aligned}
$$

with $\quad \psi_{0}(+1)-\Psi(-1)=-1$,

$$
\begin{aligned}
& \psi_{0, \mathrm{y}}=0, \psi_{0, \mathrm{x}}=0 \\
& \theta_{o}=1, C_{0}=0 \quad \text { on } \quad y=-1 \\
& \theta_{o}=\operatorname{Sin}(x+\gamma t), C_{0}=1 \quad \text { on } y=1
\end{aligned}
$$

and to the first order are

$$
\begin{aligned}
& \psi_{1, y y y y}-M_{1}^{2} \psi_{1, y y}=\frac{G}{R}\left(\theta_{1, Y y}+N C_{1, Y}\right)+\left(\psi_{0, y} \psi_{0, x y y}-\psi_{0, x} \psi_{0, y y y}\right) \\
\theta_{1, y y}= & \left(\psi_{0, x} \theta_{o, y}-\psi_{0, y} \theta_{o x}\right)+\frac{2 P E_{c} R^{2}}{G}\left(\psi_{0, y y} \cdot \psi_{1, y y}\right)+ \\
+ & \frac{2 P_{1} E_{c} M_{1}^{2}}{G}\left(\psi_{0, y} \cdot \psi_{1, y}\right) \\
& C_{1, y y}-(k S c) C_{1}=\left(\psi_{0, x} C_{o, y}-\psi_{0, y} C_{o x}\right)-\frac{S c S o}{N} \theta_{1, y y}
\end{aligned}
$$

with $\quad \psi_{1(+1)}-\psi_{1(-1)}=0$

$$
\begin{aligned}
& \psi_{1, \mathrm{y}}=0, \psi_{1, \mathrm{x}}=0 \text { at } \mathrm{y}= \pm 1 \\
& \theta_{1}( \pm 1)=0, \mathrm{C}_{1}( \pm 1)=0 \quad \text { at } \mathrm{y}= \pm 1
\end{aligned}
$$

Assuming Ec $<<1$ to be small we take the asymptotic expansions as

$$
\begin{aligned}
& \psi_{0}(x, y, t)=\psi_{00}(x, y, t)+E c \psi_{01}(x, y, t)+ \\
& \psi_{1}(x, y, t)=\psi_{10}(x, y, t)+E c \psi_{11}(x, y, t)+ \\
& \theta_{0}(x, y, t)=\theta_{00}(x, y, t)+\theta_{01}(x, y, t)+\ldots \ldots \\
& \theta_{1}(x, y, t)=\theta_{10}(x, y, t)+\theta_{11}(x, y, t)+\ldots \ldots \ldots \\
& C_{0}(x, y, t)=C_{00}(x, y, t)+C_{01}(x, y, t)+\ldots \ldots
\end{aligned}
$$




$$
C_{1}(x, y, t)=C_{10}(x, y, t)+C_{11}(x, y, t)+\ldots
$$

Substituting the expansions(22) in equations (12)-(14) and separating the like powers-of Ec we get the following

$$
\begin{aligned}
& \theta_{00, y y}=-\alpha \quad, \quad \theta_{00}(-1)=1, \theta_{00}(+1)=\operatorname{Sin} D_{1} \\
& C_{00, y y} \quad-(k S c) C_{00}=-\frac{S c S o}{N} \theta_{00, y y} \quad, \quad C_{00}(-1)=0, C_{00}(+1)=1 \\
& \psi_{00, y y y y}-M_{1}^{2} \psi_{00, y y}=\frac{G}{R}\left(\theta_{00, y}+N C_{00, y}\right), \\
& \psi_{00}(+1)-\psi_{00}(-1)=1, \psi_{00, y}=0, \psi_{00, x}=0 \text { at } y= \pm 1 \\
& \theta_{01, y y}=-\frac{P R}{G} \psi_{00, y y}^{2}-\frac{P M_{1}^{2}}{G} \psi_{00, y}^{2} \quad, \quad \theta_{01}( \pm 1)=0 \\
& C_{01, y y}-(k S c) C_{01}=-\frac{S c S o}{N} \theta_{01, y y} \quad, C_{01}(-1)=0, C_{01}(+1)=0 \\
& \psi_{01, y y y y}-M_{1}^{2} \psi_{01, y y}=\frac{G}{R}\left(\theta_{01, y}+N C_{01, y}\right) \\
& \psi_{01}(+1)-\psi_{01}(-1)=0, \psi_{01, y}=0, \psi_{01, x}=0 \text { at } y= \pm 1 \\
& \theta_{10, y y}=G P_{1}\left(\psi_{00, y} \theta_{00, x}-\psi_{00, x} \theta_{00, y}\right) \quad \theta_{10}( \pm 1)=0 \\
& C_{10, y y}-(k S c) C_{10}=S c\left(\psi_{00, y} C_{00, x}-\psi_{00, x} C_{00, y}\right)- \\
& -\frac{S c S o}{N} \theta_{01, y y} \quad C_{10}( \pm 1)=0 \\
& \psi_{10, y y y y}-M_{1}^{2} \psi_{10, y y}=\frac{G}{R}\left(\theta_{10, y}+N C_{10, Y}\right)+ \\
& +\left(\psi_{00, y} \psi_{00, x y y}-\psi_{00, x} \psi_{00, y y y}\right) \\
& \psi_{10}(+1)-\psi_{10}(-1)=0, \psi_{10, y}=0, \psi_{10, x}=0 \text { at } y= \pm 1 \\
& \theta_{11, y y}=P\left(\psi_{00, y} \theta_{01, x}-\psi_{01, x} \theta_{00, y}+\theta_{00, x} \psi_{01, y}-\theta_{01, y} \psi_{0, x}\right)- \\
& -\frac{2 P R^{2}}{G} \psi_{00, y y} \psi_{10, y y}-\frac{2 P M_{1}^{2}}{G} \psi_{00, y} \psi_{10, y}, \theta_{1}( \pm 1)=0 \\
& C_{11, y y}-(k S c) C_{11}=S c\left(\psi_{00, y} C_{01, x}-\psi_{01, x} C_{00, y}+\right. \\
& \left.+C_{00, x} \psi_{01, y}-C_{01, y} \psi_{0, x}\right)-\frac{S c S o}{N} \theta_{11, y y} \\
& \psi_{11, y y y y}-M_{1}^{2} \psi_{1, y y}=\frac{G}{R}\left(\theta_{11, y}+N C_{11, Y}\right)+\left(\psi_{00, y} \psi_{11, x y y}-\right. \\
& \left.-\psi_{00, x} \psi_{01, y y y}+\psi_{01, y} \psi_{00, x y y}-\psi_{01 . x} \psi_{00, y y y}\right) \\
& \psi_{11}(+1)-\psi_{11}(-1)=0, \psi_{11, y}=0, \psi_{11, x}=0 \text { at } y= \pm 1
\end{aligned}
$$




\section{SOLUTION OF THE PROBLEM}

Solving the equations (23)- (24) subject to the relevant boundary conditions we obtain

$$
\begin{aligned}
& \theta_{o o}(y, t)=\left(\frac{\alpha}{2}\right)\left(1-y^{2}\right)+\frac{\operatorname{Sin}\left(D_{1}\right)}{2}(y+1)+0.5(1-y) \\
& C_{00}=0.5\left(\frac{\operatorname{Sinh}\left(\beta_{1} y\right)}{\operatorname{Sinh}\left(\beta_{1}\right)}+\frac{\operatorname{Cosh}\left(\beta_{1} y\right)}{\operatorname{Cosh}\left(\beta_{1}\right)}\right)+a_{3}\left(1-\frac{\operatorname{Cosh}\left(\beta_{1} y\right)}{\operatorname{Cosh}\left(\beta_{1}\right)}\right) \\
& \psi_{o o}(y, t)=a_{11} \operatorname{Cosh}\left(M_{1} y\right)+a_{12} \operatorname{Sinh}\left(M_{1} y\right)+a_{13} y+a_{14}+\phi_{1}(y) \\
& \phi_{1}(y)=a_{6} y+a_{7} y^{2}++a_{8} y^{3}-a_{10} \operatorname{Ch}\left(\beta_{1} y\right)-a_{11} \operatorname{Sh}\left(\beta_{1} y\right) \\
& \theta_{01}(y, t)=0.5 a_{19}\left(y^{2}-1\right)+\frac{a_{20}}{4 M_{1}^{2}}\left(\operatorname{Ch}\left(2 M_{1} y\right)-\operatorname{Ch}\left(2 M_{1}\right)\right)+\frac{a_{21}}{4 \beta_{1}^{2}}\left(\operatorname{Ch}\left(2 \beta_{1} y\right)-\right. \\
& \left.-C h\left(2 \beta_{1}\right)\right)+\frac{a_{22}}{M_{1}^{2}}\left(C h\left(M_{1} y\right)-C h\left(M_{1}\right)\right)+a_{36}\left(y \operatorname{Sh}\left(M_{1} y\right)-\right. \\
& \left.-\operatorname{Sh}\left(M_{1}\right)\right)+a_{37}\left(y \operatorname{Sh}\left(\beta_{1} y\right)-\operatorname{Sh}\left(\beta_{1}\right)\right)+\frac{a_{23}}{\beta_{1}^{2}}\left(\left(\operatorname{Ch}\left(\beta_{1} y\right)-\operatorname{Ch}\left(\beta_{1}\right)\right)+\frac{a_{24}}{M_{1}^{2}}\left(y \operatorname{Sh}\left(M_{1} y\right)-\right.\right. \\
& \left.-S h\left(M_{1}\right)\right)-\frac{2 a_{24}}{M_{1}^{3}}\left(a_{40}\left(C h\left(M_{1} y\right)-\operatorname{Ch}\left(M_{1}\right)\right)+\frac{a_{25}}{\beta_{2}^{2}}\left(C h\left(\beta_{2} y\right)-\operatorname{Ch}\left(\beta_{2}\right)\right)+\frac{a_{26}}{\beta_{3}^{2}}\left(\operatorname{Ch}\left(\beta_{3} y\right)\right.\right. \\
& \left.\left.-C h\left(\beta_{3}\right)\right)+\frac{a_{27}}{12}\left(y^{4}-1\right)-C h\left(\beta_{3}\right)\right)+\frac{a_{28}}{30}\left(y^{6}-1\right) \\
& C_{01}(y, t)=a_{31}\left(1-\frac{C h\left(\beta_{1} y\right)}{C h\left(\beta_{1}\right)}\right)+a_{32}\left(y-\frac{\operatorname{Sh}\left(\beta_{1} y\right)}{\operatorname{Sh}\left(\beta_{1}\right)}\right)+a_{33}\left(y^{2}-\frac{C h\left(\beta_{1} y\right)}{C h\left(\beta_{1}\right)}\right)+ \\
& +a_{34}\left(y^{4}-\frac{C h\left(\beta_{1} y\right)}{C h\left(\beta_{1}\right)}\right)+a_{354} a_{33}\left(y^{6}-\frac{C h\left(\beta_{1} y\right)}{C h\left(\beta_{1}\right)}\right)+a_{36}\left(C h\left(2 M_{1} y\right)-\right. \\
& \left.-C h\left(2 M_{1}\right) \frac{C h\left(\beta_{1} y\right)}{C h\left(\beta_{1}\right)}\right)+a_{37}\left(C h\left(2 \beta_{1} y\right)--C h\left(2 \beta_{1}\right) \frac{C h\left(\beta_{1} y\right)}{C h\left(\beta_{1}\right)}\right)+ \\
& +a_{38}\left(C h\left(M_{1} y\right)-\operatorname{Ch}\left(M_{1}\right) \frac{\operatorname{Ch}\left(\beta_{1} y\right)}{\operatorname{Ch}\left(\beta_{1}\right)}\right)+a_{39}\left(y \operatorname{Sh}\left(\beta_{1} y\right)-\right. \\
& \left.-\operatorname{Sh}\left(\beta_{1}\right) \frac{\operatorname{Sh}\left(\beta_{1} y\right)}{\operatorname{Sh}\left(\beta_{1}\right)}\right)+a_{40}\left(y^{2} \operatorname{Ch}\left(M_{1} y\right)-\operatorname{Ch}\left(M_{1}\right) \frac{\operatorname{Ch}\left(\beta_{1} y\right)}{\operatorname{Ch}\left(\beta_{1}\right)}\right)+ \\
& +a_{41}\left(C h\left(\beta_{2} y\right)-C h\left(\beta_{2}\right) \frac{\operatorname{Ch}\left(\beta_{1} y\right)}{\operatorname{Ch}\left(\beta_{1}\right)}\right)+a_{42}\left(\operatorname{Ch}\left(\beta_{3} y\right)-\operatorname{Ch}\left(\beta_{3}\right) \frac{\operatorname{Ch}\left(\beta_{1} y\right)}{\operatorname{Ch}\left(\beta_{1}\right)}\right) \\
& \psi_{01}(y, t)=a_{58}+a_{57} y+a_{55} \operatorname{Ch}\left(M_{1} y\right)+a_{56} \operatorname{Sh}\left(2 \beta_{2} y\right)+\phi_{2}(y) \\
& \phi_{2}(y)=a_{43}+a_{44} y+a_{45} y^{3}+a_{46} y^{5}+a_{47} \operatorname{Sh}\left(2 M_{1} y\right)+a_{48} \operatorname{Sh}\left(2 \beta_{1} y\right)+a_{49} \operatorname{Sh}\left(M_{1} y\right)+ \\
& +a_{50} y \operatorname{Ch}\left(M_{1} y\right)+a_{51} y \operatorname{Ch}\left(\beta_{1} y\right)+a_{52} y^{2} \operatorname{Sh}\left(M_{1} y\right)+a_{53} \operatorname{Sh}\left(\beta_{2} y\right)+a_{54} \operatorname{Sh}\left(\beta_{3} y\right)
\end{aligned}
$$




$$
\begin{aligned}
& \theta_{10}(y, t)=a_{77} y^{2}+a_{78} y^{3}+a_{79} y^{4}+a_{80} y^{5}+a_{817} C h\left(\left(\beta_{1} y\right)+a_{82} \operatorname{Sh}\left(\beta_{1} y\right)+\right. \\
& +a_{83} \operatorname{Ch}\left(M_{1} y\right)+a_{84} \operatorname{Sh}\left(M_{1} y\right)+a_{85} y \operatorname{Ch}\left(M_{1} y\right)+a_{86} y \operatorname{Sh}\left(M_{1} y\right)+ \\
& +a_{87} y C h\left(\beta_{1} y\right)+a_{88} y \operatorname{Sh}\left(\beta_{1} y\right)+a_{89} y+a_{90} \\
& C_{10}(y, t)=b_{14} \operatorname{Ch}\left(\beta_{1} y\right)+b_{15} \operatorname{Sh}\left(\beta_{1} y\right)+\phi_{3}(y) \\
& \phi_{3}(y)=b_{1}+b_{2} \operatorname{Ch}\left(2 \beta_{2} y\right)+b_{35} \operatorname{Sh}\left(2 \beta_{1} y\right)+\left(b_{4} y+b_{6} y^{2}+b_{8} y^{3}\right) \operatorname{Ch}\left(\beta_{1} y\right) \\
& +\left(b_{5} y+b_{7} y^{2}+b_{9} y^{3}\right) \operatorname{Sh}\left(\beta_{1} y\right)+b_{10} \operatorname{Sh}\left(\beta_{2} y\right)+b_{11} \operatorname{Sh}\left(\beta_{3} y\right)+ \\
& +b_{12} \operatorname{Ch}\left(\beta_{2} y\right)+b_{13} \operatorname{Ch}\left(\beta_{3} y\right) \\
& \psi_{10}=b_{80} \operatorname{Ch}\left(M_{1} y\right)+b_{81} \operatorname{Sh}\left(M_{1} y\right)+b_{82} y+b_{83}+\phi_{4}(y) \\
& \phi_{4}(y)=b_{53} y^{2}+b_{54} y^{3}+b_{55} y^{4}+b_{56} y^{5}+b_{57} y^{6}+\left(b_{58}+b_{64} y+b_{66} y^{2}+b_{71} y^{3}+\right. \\
& \left.+b_{73} y^{4}\right) \operatorname{Sh}\left(\beta_{1} y\right)+\left(b_{59}+b_{65} y+b_{67} y^{2}+b_{70} y^{3}+b_{72} y^{4}\right) C h\left(\beta_{1} y\right)+ \\
& +\left(b_{60} y+b_{63} y^{2}+d_{46} y^{3}\right) \operatorname{Ch}\left(M_{1} y\right)++\left(b_{61} y+b_{62} y^{2}\right) \operatorname{Sh}\left(M_{1} y\right)+ \\
& +b_{75} y C h\left(2 M_{1} y\right)+b_{74} y \operatorname{Sh}\left(2 M_{1} y\right)+b_{76} \operatorname{Sh}\left(\beta_{2} y\right)+b_{77} \operatorname{Sh}\left(\beta_{3} y\right)+b_{78} \operatorname{Ch}\left(\beta_{2} y\right) \\
& +b_{78} \operatorname{Ch}\left(\beta_{3} y\right)
\end{aligned}
$$

where $a_{1}, a_{2}$

$a_{105}, b_{1}, b_{2}$

are constants involving the governing

parameters.

\section{NUSSELT NUMBER and SHERWOOD NUMBER}

The local rate of heat transfer coefficient (Nusselt number $\mathrm{Nu}$ ) on the walls has been calculated using the formula $N u=\frac{1}{\theta_{m}-\theta_{w}}\left(\frac{\partial \theta}{\partial y}\right)_{y= \pm 1}$ and the corresponding expressions are

$$
(N u)_{y=+1}=\frac{\left(d_{2}+E c d_{4}+\delta d_{6}\right)}{\left(\theta_{m}-\operatorname{Sin}\left(D_{1}\right)\right.},(N u)_{y=-1}=\frac{\left(d_{3}+E c d_{5}+\delta d_{7}\right)}{\left(\theta_{m}-1\right)}, \theta_{m}=d_{8}+E c d_{9}+\delta d_{10}
$$

The local rate of mass transfer coefficient( Sherwood number)( Sh) on the walls has been calculated using the formula $S h=\frac{1}{C_{m}-C_{w}}\left(\frac{\partial C}{\partial y}\right)_{y= \pm 1}$ and the corresponding expressions are

$$
(S h)_{y=+1}=\frac{\left(d_{11}+E c d_{13}+\delta d_{15}\right)}{\left(C_{m}-1\right)},(S h)_{y=-1}=\frac{\left(d_{12}+E c d_{14} \delta d_{16}\right)}{\left(C_{m}\right)}, C_{m}=d_{17}+E c d_{18}+\delta d_{19}
$$

where $\mathrm{d}_{1}, \ldots \ldots \ldots . . . \mathrm{d}_{19}$ constants in involving parameters..

\section{DISCUSSION OF THE RESULTS}

In this analysis we investigate the effect of chemical reaction thermo diffusion and dissipation on unsteady convective heat and mass transfer flow in a vertical channel on whose walls a travelling wave is imposed. The equations governing the non linear coupled equations 
have been solved by employing the perturbation technique with the aspect ratio $\delta$ as a perturbation parameter.

The axial velocity $(\mathrm{u})$ is shown in figures $1-5$.For different values of Sc \& So, N,k, Ec, \& $\mathrm{x}+\gamma \mathrm{t}$. It is found that the actual axial flow is in the vertically upward direction and therefore $\mathrm{u}<0$ represents the reversal flow. It is found that lesser the molecular diffusivity larger the velocity in the entire flow region $(\mathrm{Sc})$. $|\mathrm{u}|$ enhances with increase in $\mathrm{S}_{\mathrm{o}}>0$ and depreciates with $\left|S_{\mathrm{o}}\right|$ (fig .1). Fig.2 represents u with buoyancy ratio N. It is found that when the molecular buoyancy force dominates over the thermal buoyancy force, the axial velocity enhances when the buoyancy forces act in the same direction and for the forces act in opposite direction $u$ depreciates in the flow region. Fig 3 represents $u$ with the chemical reaction parameter $\mathrm{k}$. It is found that the axial velocity depreciates with increase in chemical reaction parameter $\mathrm{k}$. The variation of $\mathrm{u}$ with Eckert number Ec is shown in fig 4.It is found that higher the dissipative heat smaller the velocity in the flow region. From fig 5 we notice that the axial velocity enhances with increase in the phase $x+\gamma t \leq \pi$ and depreciates with higher $\mathrm{x}+\gamma \mathrm{t} \geq 2 \pi$.

The secondary velocity (v) is shown in figures 6-10 for different parametric values. Fig 6 represents $\mathrm{v}$ with $\mathrm{Sc}$ and $\mathrm{S}_{\mathrm{o}}$ it is found lesser the molecular diffusivity smaller $|\mathrm{v}|$ in the left half and larger the right half and for further lowering of the molecular diffusivity larger in the left half and smaller in the right half, an increase in $S_{0}>0$ reduces $|v|$ in the left half and enhances in the right half while a reversed effect is observed in the behavior $|v|$ with increase in $\left|S_{\circ}\right|$. From fig 7 we notice that the magnitude of $v$ enhances with increase in $N>0$ and depreciates with $|\mathrm{N}|$ in the entire flow region. Fig 8 represents $\mathrm{v}$ with chemical reaction parameter $\mathrm{k}$. It is found that the $|\mathrm{v}|$ enhances in the left half and reduces in the right half with increase in $\mathrm{k}$. Fig 9 represents $\mathrm{v}$ with Ec. It is observed that higher the dissipative heat larger $|v|$ in the left half and smaller in the right half. Fig 10 represents $V$ with the phase $x+\gamma t$ of the boundary temperature $|v|$ enhances with increase in $x+\gamma t \leq \pi$ and depreciates with higher $\mathrm{x}+\gamma \mathrm{t}=2 \pi$.

The non- dimensional temperature $(\theta)$ is shown in figures 11-16 for different parametric values. Fig 11 represents $\theta$ with heat source parameter $\alpha$. From the profiles we notice that the actual temperature enhances with increase in strength of the heat and depreciates with that of heat sink. Fig 12 represents $\theta$ with Soret parameter $S_{0}$ it is found that the actual temperature depreciates with increase in $S_{0}>0$ and enhances with increase in $\left|S_{0}\right|$ $(<0)$.Fig 13 represents $\theta$ with buoyancy ratio $\mathrm{N}$. It is found that when the molecular buoyancy force dominates over the thermal buoyancy force. The actual temperature enhances when the buoyancy forces act in the same direction and for the forces act in opposite direction it depreciates in the flow region. The variation of $\theta$ with chemical reaction $\mathrm{K}$ is shown in fig 14.It is found that the actual temperature reduces in degenerating chemical reaction case. The variation of $\theta$ with Ec is shown fig 15. It is observe that higher the dissipative heat larger the actual temperature. Fig 16 represents $\theta$ with phase $x+\gamma t$ of the boundary temperature. It is found that the actual temperature enhances with $x+\gamma t \leq \pi / 2$ and depreciates with higher $\mathrm{x}+\gamma \mathrm{t}=\Pi$ and again enhances with still higher $\mathrm{x}+\gamma \mathrm{t}=2 \pi$.

The concentration distribution (c) is shown in figures 17-22 for different parametric values. We follow the convention that the non-dimensional concentration is positive or negative according as the actual concentration is greater-lesser than $\mathrm{C} 2$. With respect to Sc (Fig.17). we notice that lesser the molecular diffusivity larger the actual concentration and for further lowering of the diffusivity smaller the actual concentration. With respect to $S_{0}$, we find that the actual concentration depreciates with $S_{0}>0$ and enhances with $\left|S_{0}\right|$ (fig.18). From fig 19 it is found that the actual concentration depreciates with $|N|$ irrespective of the directions of buoyancy forces. Fig 20 represents $\mathrm{C}$ with chemical reaction 
parameter $\mathrm{K}$. It is found that the actual concentration depreciates with increase in $\mathrm{K} \leq 1.5$ and depreciates with higher $\mathrm{K} \geq 2.5$.Fig 21 represents $\mathrm{C}$ with $\mathrm{Ec}$. It is found that higher the dissipative force smaller the actual concentration in the flow region. Fig 22 represents $C$ with phase $\mathrm{x}+\gamma \mathrm{t}$ of the boundary temperature. It is found that the actual concentration enhances with increase $x+\gamma t \leq \pi / 2$ and depreciates with higher $x+\gamma t \geq \pi$.

The rate heat transfer at the boundaries at $y= \pm 1$ are shown in tables 1-6 for different parametric values, variation of $\mathrm{Nu}$ with heat source parameter $\alpha$. It is found that an increase in the strength of the heat source $\alpha$ enhances $|\mathrm{Nu}|$ at $\mathrm{y}=+1$ and reduces at $\mathrm{y}=-1$ and for higher $\alpha \geq 6$ we notice a depreciation at $y= \pm 1$. $|\mathrm{Nu}|$ experiences an enhancement with increase in the strength of the heat sink. With respect to the Schmidt number Sc we find that lesser the molecular diffusivity smaller $|\mathrm{Nu}|$ in the heating case and larger $|\mathrm{Nu}|$ in the cooling case. The variation of $\mathrm{Nu}$ with Soret parameter $\mathrm{S}_{0}$ shows that $|\mathrm{Nu}|$ enhances with increase in $\left|S_{0}\right|$ at $\mathrm{y}=+1$ and at $\mathrm{y}=-1$ the rate of heat transfer reduces with $\mathrm{S}_{0}=0$ and enhances $\left|S_{0}\right|$ for $\mathrm{G}>0$ while for $\mathrm{G}<0|\mathrm{Nu}|$ enhances with $\mathrm{S}_{0}$ and reduces $\left|\mathrm{S}_{0}\right|$. The variation of $\mathrm{Nu}$ with buoyancy ratio $\mathrm{N}$ shows that when the molecular buoyancy force dominates over the thermal buoyancy force, the rate of heat transfer enhances irrespective of the directions of the buoyancy forces. At $y=-$ 1 rate of heat transfer depreciates in the heating case and enhances in the cooling case with increase in $\mathrm{G}>0$ and reduces with increase in $|\mathrm{N}|$ for all $\mathrm{G}$. We noticed that higher the radiative heat flux larger $|\mathrm{Nu}|$ at both the walls. With respect to chemical reaction parameter $\mathrm{K}$. we find that the rate of heat transfer at $\mathrm{y}=+1$ deprecites with $\mathrm{K} \leq 1.5$ and enhances with higher $K \geq 2.5$. While at $y=-1|\mathrm{Nu}|$ enhances with increase in $\mathrm{K}$ for all $\mathrm{G}$. The variation of $\mathrm{Nu}$ with Eckert number Ec shows that higher the dissipative heat larger $|\mathrm{Nu}|$ at $\mathrm{y}=+1$ and smaller at $y=-1$. An increase in the phase $x+\gamma t \leq \pi / 2$ reduces $|N u|$ and enhances with higher $x+\gamma t \geq \pi$ at both the walls.

The rate of mass transfer (Sherwood number) at $y= \pm 1$ is shown in tables 7-12 for different parametric values. With respect to heat source parameter, $|\mathrm{Sh}|$ enhances at $y= \pm 1$ with increase in $\alpha \geq 0$ while it enhances at $y=+1$ and depreciates at $y=-1$ with $|\alpha| \leq 4$ and depreciates at both the walls with higher $|\alpha| \geq 6$.The variation of Sh with Sc shows that lesser the molecular diffusivity larger $|\mathrm{Sh}|$ at $\mathrm{y}= \pm 1$.The variation of $\mathrm{Sh}$ with Soret parameter $\mathrm{S}_{0}$ exhibits that $|\mathrm{Sh}|$ at $\mathrm{y}=+1$ enhances with increase in $\left|\mathrm{S}_{0}\right|$, while at $\mathrm{y}=-1|\mathrm{Sh}|$ depreciates with $\left|S_{0}\right|>0$ and enhances with $\left|S_{0}\right|$ in the heating case and in the cooling case it enhances with $S_{0}>0$ and reduces with $\left|\mathrm{S}_{0}\right|$. With respect to the Buoyancy ratio $\mathrm{N}$, we find that when the molecular Buoyancy force dominates over the thermal Buoyancy force. The rate of mass transfer enhances at $\mathrm{y}=+1$ and depreciates at $\mathrm{y}=-1$ when the Buoyancy forces act in the same direction and for the forces act in opposite direction $|\mathrm{Sh}|$ depreciates at $\mathrm{y}=+1$ and enhances at $\mathrm{y}=-1$. The revels that higher the radiative heat flux smaller $|\mathrm{Sh}|$ at $\mathrm{y}=+1$ and larger at $\mathrm{y}=-1$. The chemical reaction parameter $K$, it is observed that rate of mass transfer at $y=+1$ depreciates with $K \leq 2.5$ and enhances with higher $K \geq 3.5$, while at $y=-1$ it enhances with $K$ for all $G$. The variation of Sh with Eckert number Ec shows that higher the dissipative heat smaller $|\mathrm{Sh}|$ at $\mathrm{y}=+1$ and larger at $y=-1$.The variation of $S h$ with phase $x+\gamma t$ shows that $|S h|$ depreciates at $y=+1$ and enhances at $y=-1$ with increase in $x+\gamma t \leq \pi$, while for higher $x+\gamma t \geq 2 \pi$, $\mid$ Sh $\mid$ enhances at $y=+1$ and reduces at $\mathrm{y}=-1$. 


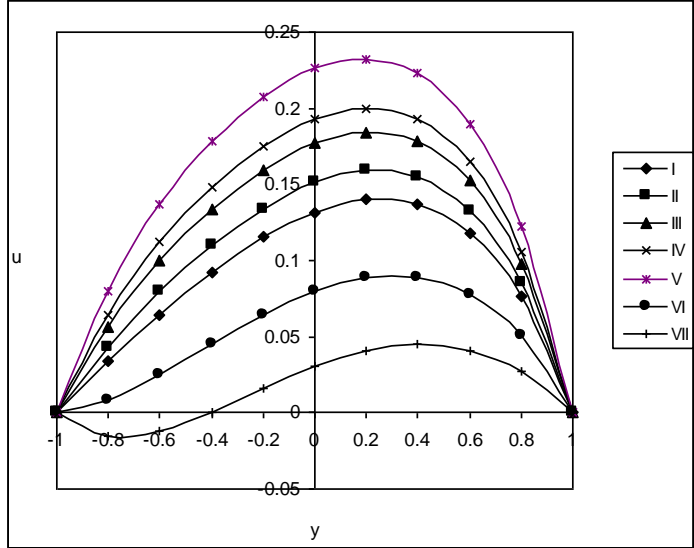

Fig. 1 : Variation of $\mathrm{u}$ with $\mathrm{Sc}, \mathrm{S}_{0}$

$\begin{array}{cccccccc} & \text { I } & \text { II } & \text { III } & \text { IV } & \text { V } & \text { VI } & \text { VII } \\ \text { Sc } & 0.24 & 0.6 & 1.3 & 2.01 & 1.3 & 1.3 & 1.3 \\ \mathrm{~S}_{0} & 0.5 & 0.5 & 0.5 & 0.5 & 1 & -5 & -1\end{array}$

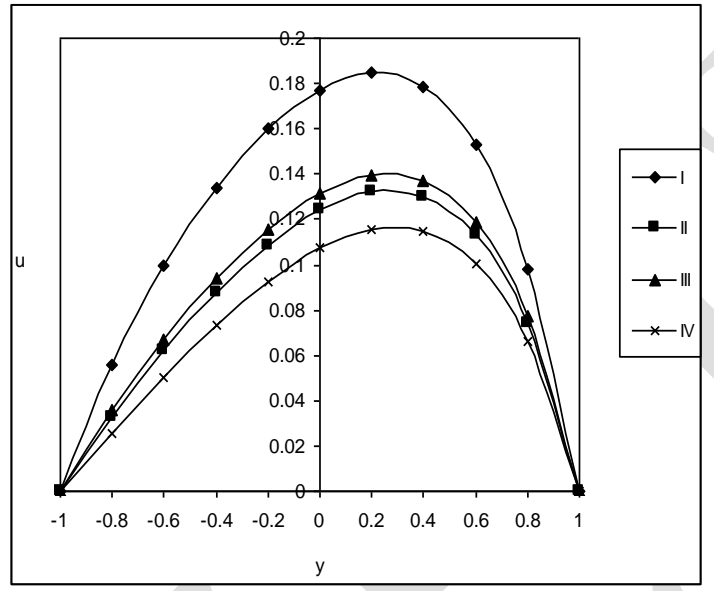

Fig. 3 : Variation of $\mathrm{u}$ with $\mathrm{k}$

$\begin{array}{llll}\mathrm{I} & \text { II } & \text { III } & \text { IV } \\ 0.5 & 2.15 & 2.5 & 3.5\end{array}$

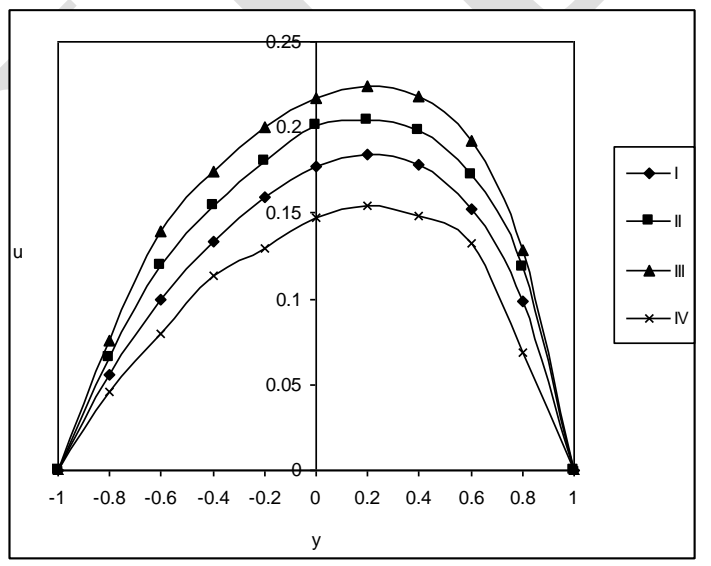

Fig. 5 : Variation of $\mathrm{u}$ with $\mathrm{x}+\gamma \mathrm{t}$

$\begin{array}{lllll} & \text { I } & \text { II } & \text { III } & \text { IV } \\ \mathrm{x}+\gamma \mathrm{t} & \pi / 4 & \pi / 2 & \pi & 2 \pi\end{array}$

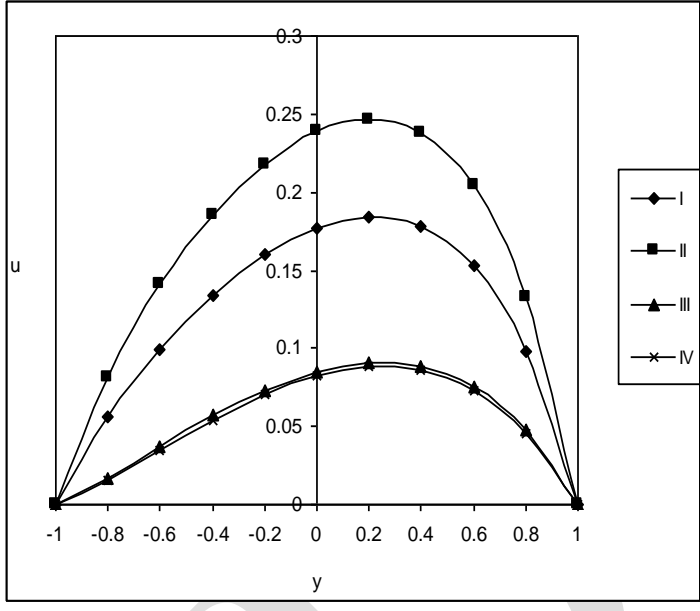

Fig. 2: Variation of u with $\mathrm{N}$

$\begin{array}{ccccc} & \text { I } & \text { II } & \text { III } & \text { IV } \\ \text { N } & 1 & 2 & -0.5 & -0.8\end{array}$

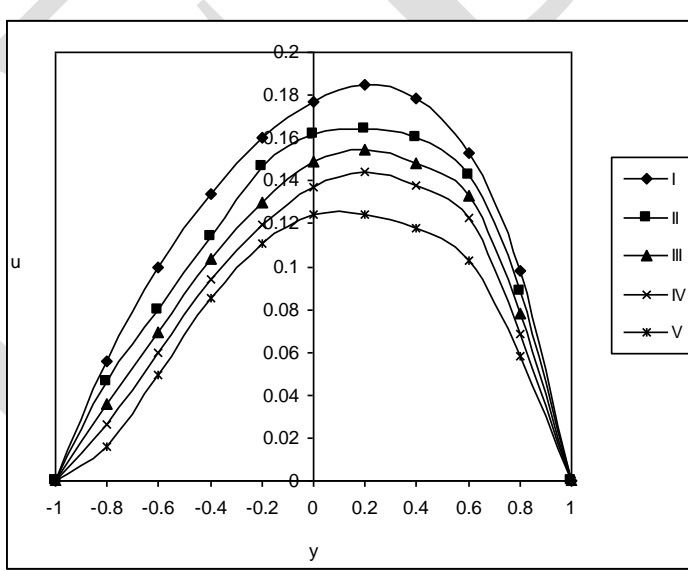

Fig. 4 : Variation of $\mathrm{u}$ with Ec $\begin{array}{cccccc} & \text { I } & \text { II } & \text { III } & \text { IV } & \text { V } \\ \text { Ec } & 0.01 & 0.03 & 0.05 & 0.07 & 0.09\end{array}$

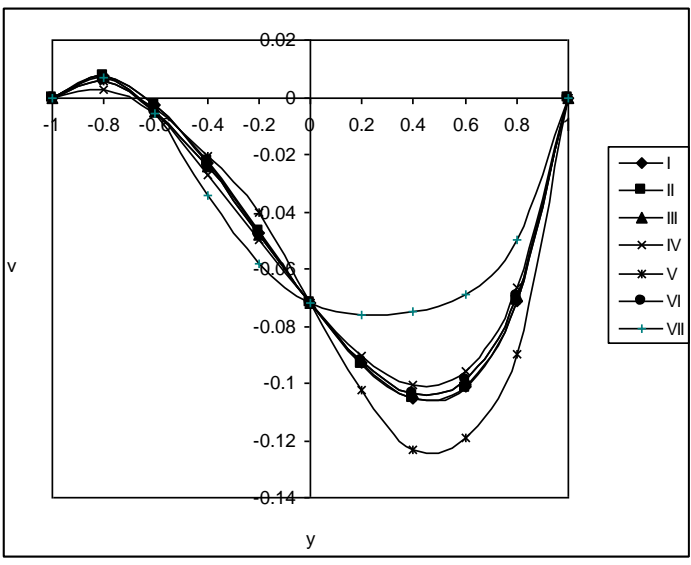

Fig. 6 : Variation of $\mathrm{v}$ with $\mathrm{Sc}, \mathrm{S}$

$\begin{array}{llllllll} & \text { I } & \text { II } & \text { III } & \text { IV } & \text { V } & \text { VI } & \text { VII } \\ \text { Sc } & 0.24 & 0.6 & 1.3 & 2.01 & 1.3 & 1.3 & 1.3\end{array}$

$\begin{array}{llllllll}\mathrm{S}_{0} & 0.5 & 0.5 & 0.5 & 0.5 & 1 & -5 & -1\end{array}$ 


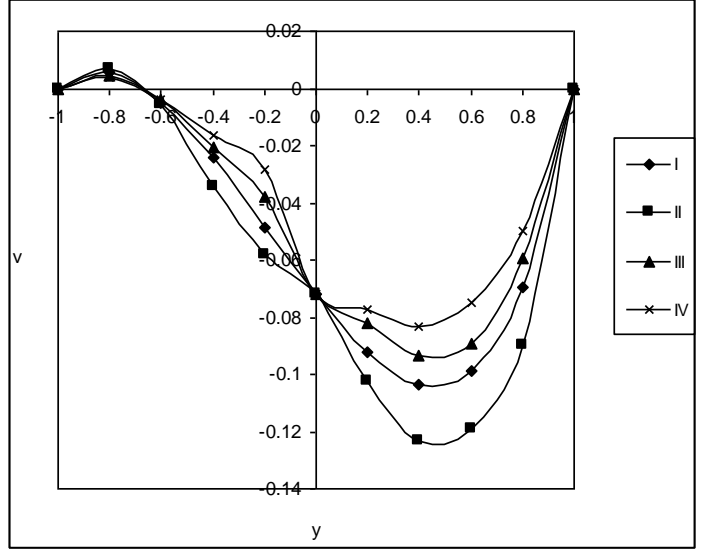

Fig. 7 : Variation of $\mathrm{v}$ with $\mathrm{N}$

$\begin{array}{ccccc} & \text { I } & \text { II } & \text { III } & \text { IV } \\ \mathrm{N} & 1 & 2 & -0.5 & -0.8\end{array}$

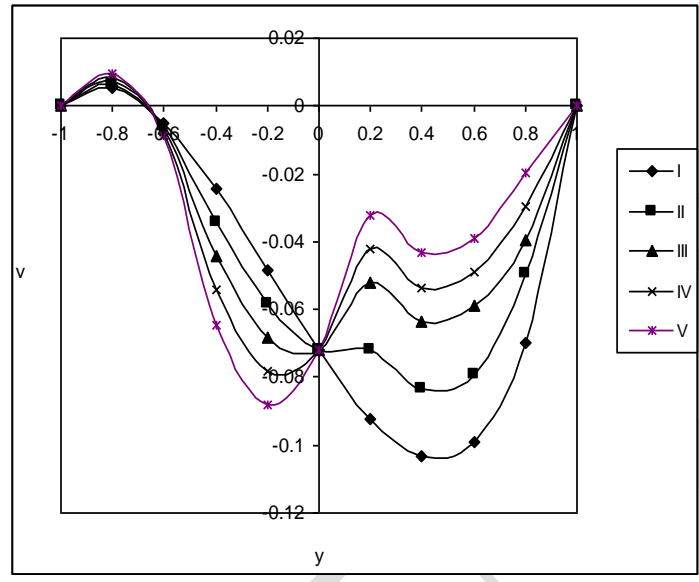

Fig. 9 : Variation of $\mathrm{v}$ with $\mathrm{Ec}$ $\begin{array}{cccccc} & \text { I } & \text { II } & \text { III } & \text { IV } & \text { V } \\ \text { Ec } & 0.01 & 0.03 & 0.05 & 0.07 & 0.09\end{array}$

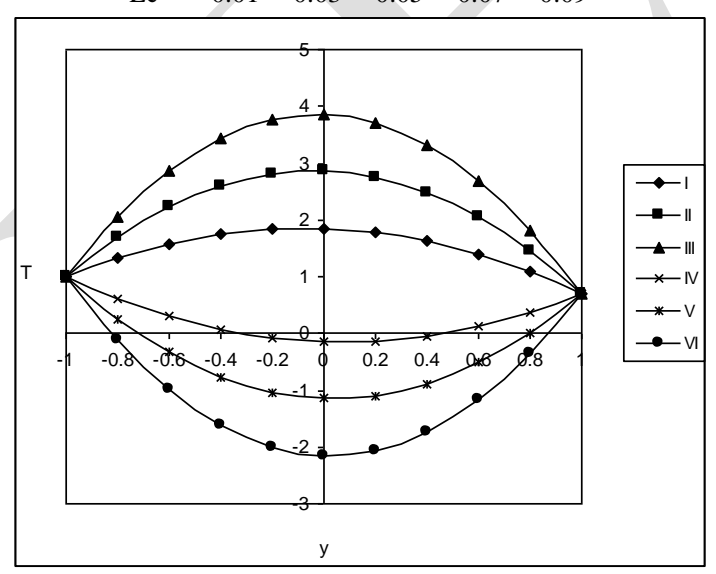

Fig. 11 : Variation of $\mathrm{T}$ with $\alpha$

$\begin{array}{lllllll} & \text { I } & \text { II } & \text { III } & \text { IV } & \text { V } & \text { VI } \\ \alpha & 2 & 4 & 6 & -2 & -4 & -6\end{array}$

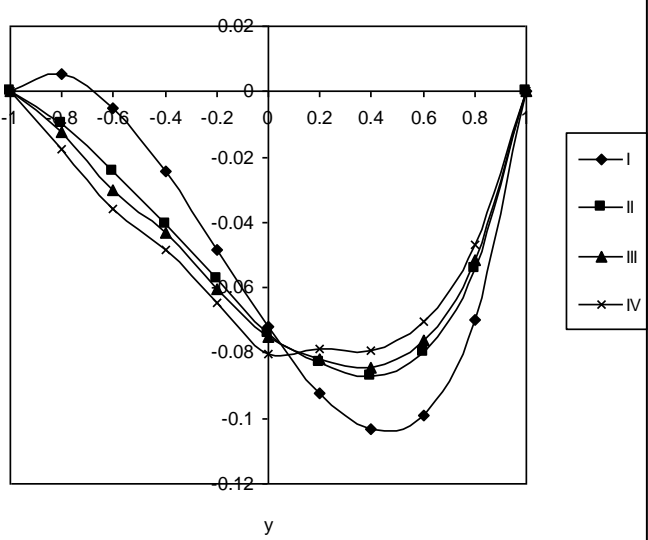

Fig. 8 : Variation of $\mathrm{v}$ with $\mathrm{k}$

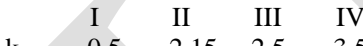

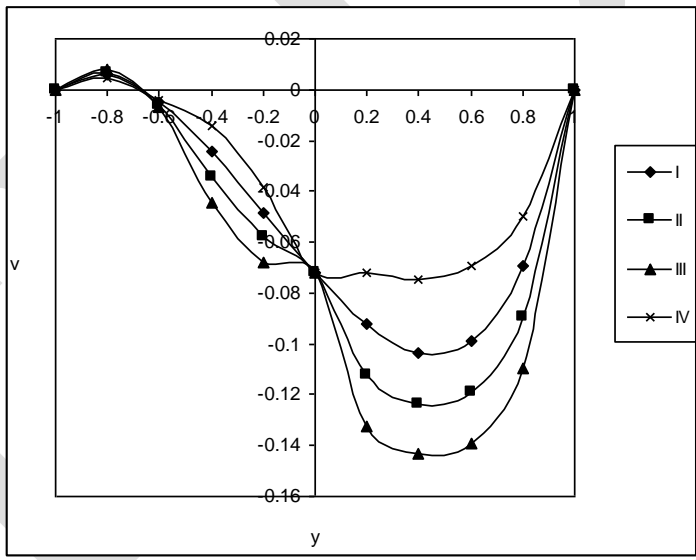

Fig. 10 : Variation of $\mathrm{v}$ with $\mathrm{x}+\gamma \mathrm{t}$

$\begin{array}{rrrr}\text { I } & \text { II } & \text { III } & \text { IV } \\ \mathrm{x}+\gamma \mathrm{t} & \pi / 4 & \pi / 2 & \pi\end{array}$

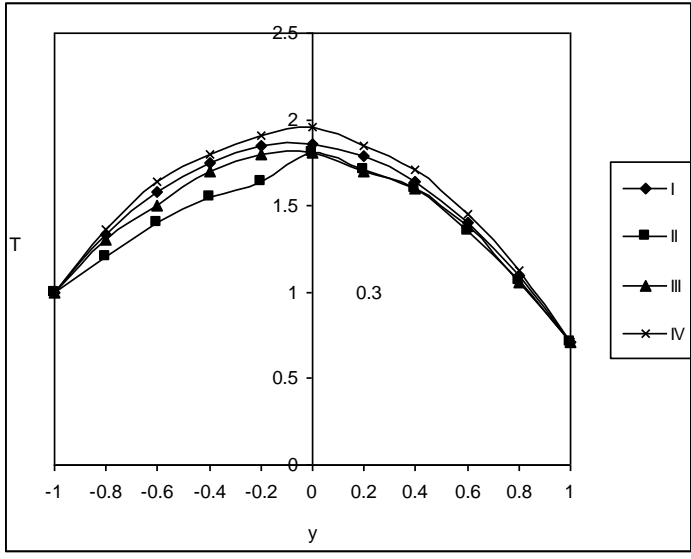

Fig. 12 : Variation of $\mathrm{T}$ with $\mathrm{S}_{0}$

$\begin{array}{llll}\text { I } & \text { II } & \text { III } & \text { IV } \\ 0.5 & 1 & -5 & -1\end{array}$ 


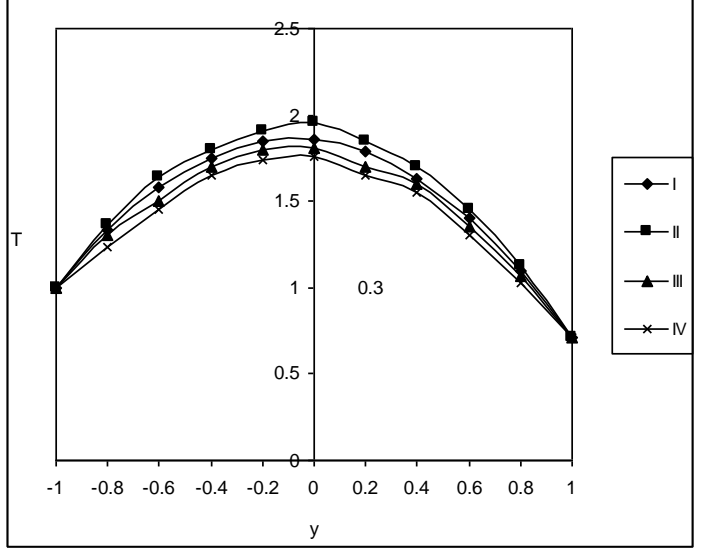

Fig. 13 : Variation of $\mathrm{T}$ with $\mathrm{N}$

$\begin{array}{lllrr} & \text { I } & \text { II } & \text { III } & \text { IV } \\ \mathrm{N} & 1 & 2 & -0.5 & -0.55\end{array}$

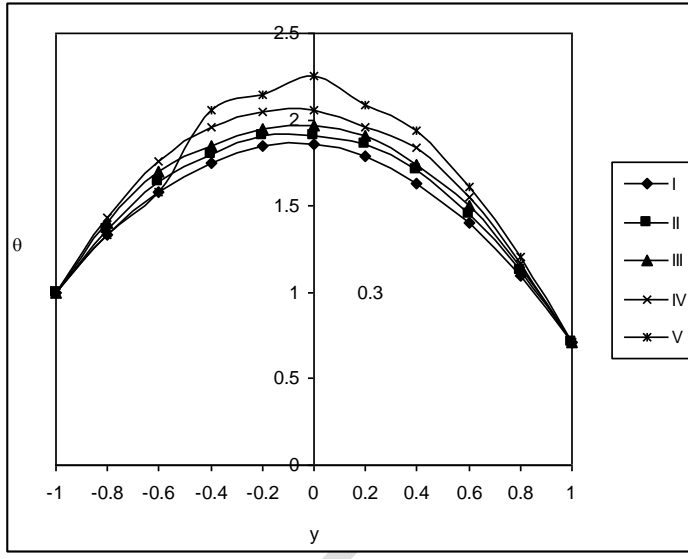

Fig. 15 : Variation of $\mathrm{T}$ with Ec $\begin{array}{cccccc} & \text { I } & \text { II } & \text { III } & \text { IV } & \text { V } \\ \text { Ec } & 0.01 & 0.03 & 0.05 & 0.07 & 0.09\end{array}$

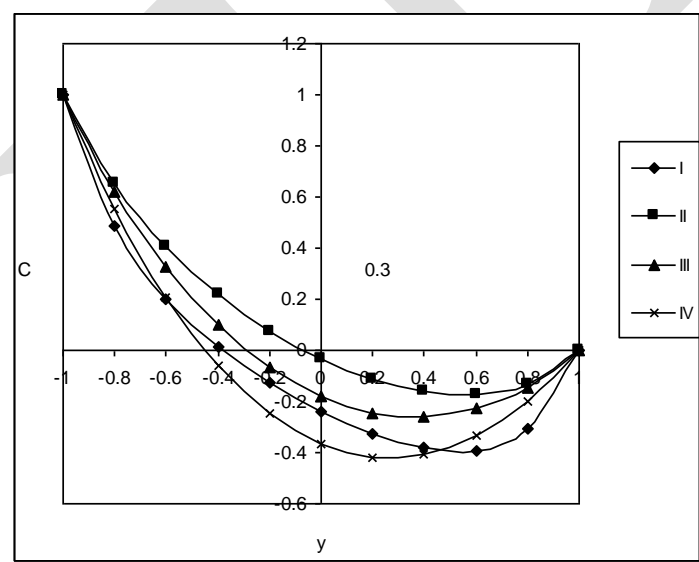

Fig. 17 : Variation of $\mathrm{C}$ with $\mathrm{Sc}$

Sc $\quad 0.24 \quad 0.6 \quad 1.3 \quad 2.01$

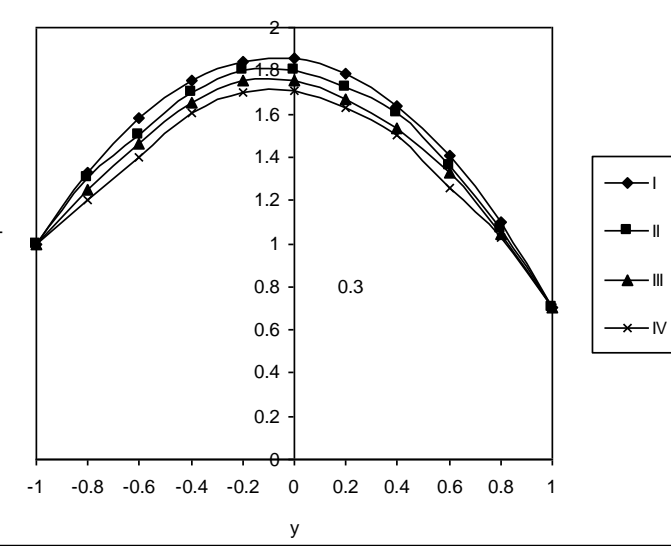

Fig.14 : Variation of $\mathrm{T}$ with $\mathrm{k}$

$\begin{array}{llll}\text { I } & \text { II } & \text { III } & \text { IV }\end{array}$

$\begin{array}{lllll}\mathrm{k} & 0.5 & 2.15 & 2.5 & 3.5\end{array}$

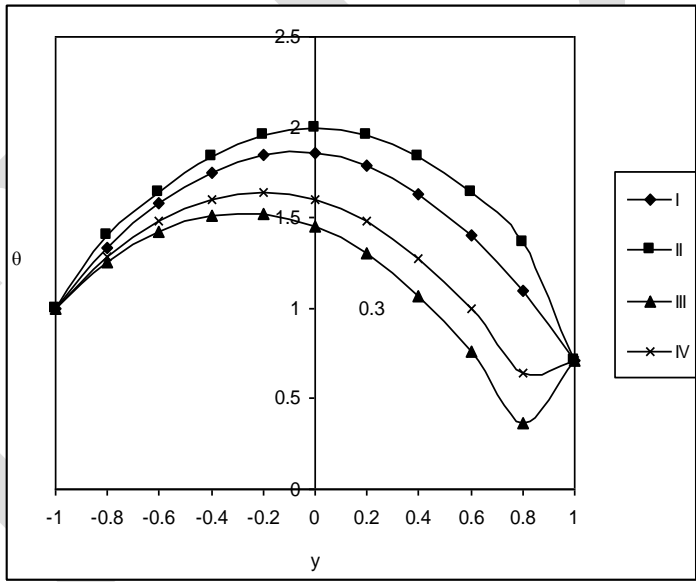

Fig. 16 : Variation of $\mathrm{T}$ with $\mathrm{x}+\gamma \mathrm{t}$

I II III IV

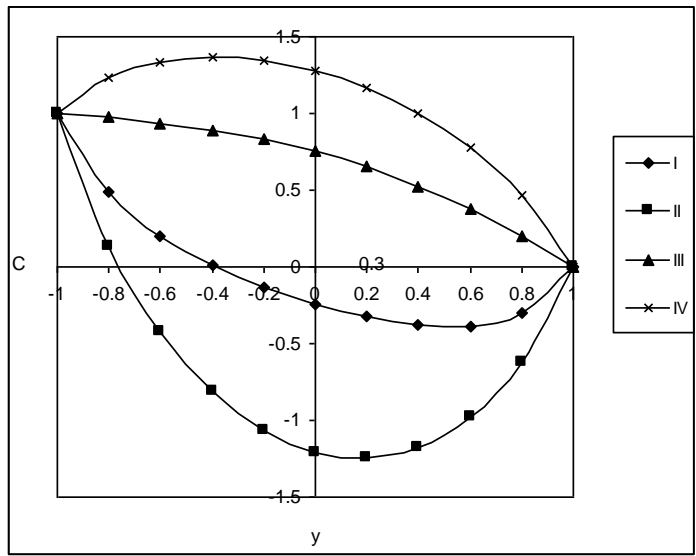

Fig. 18 : Variation of $\mathrm{C}$ with $\mathrm{S}_{0}$

$\begin{array}{llll}1 & \text { II } & \text { III } & \text { IV } \\ 0.5 & 1 & -5 & \end{array}$ 


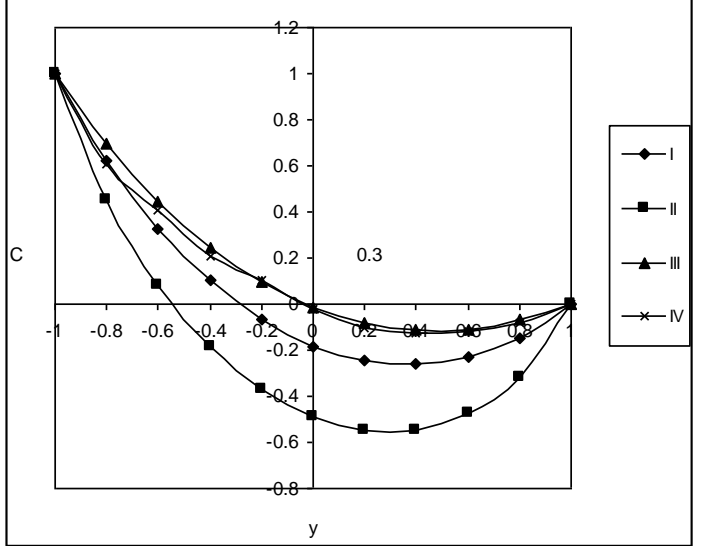

Fig. 19 : Variation of $\mathrm{C}$ with $\mathrm{N}$

\begin{tabular}{lllrr}
$\mathrm{N}$ & \multicolumn{1}{c}{ I } & II & III & IV \\
& 1 & 2 & -0.5 & -0.55
\end{tabular}

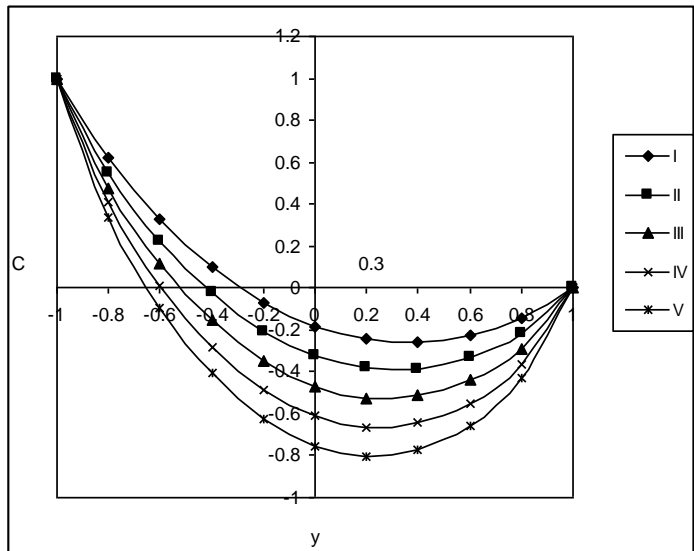

Fig. 21 : Variation of $\mathrm{C}$ with Ec

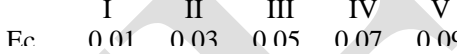

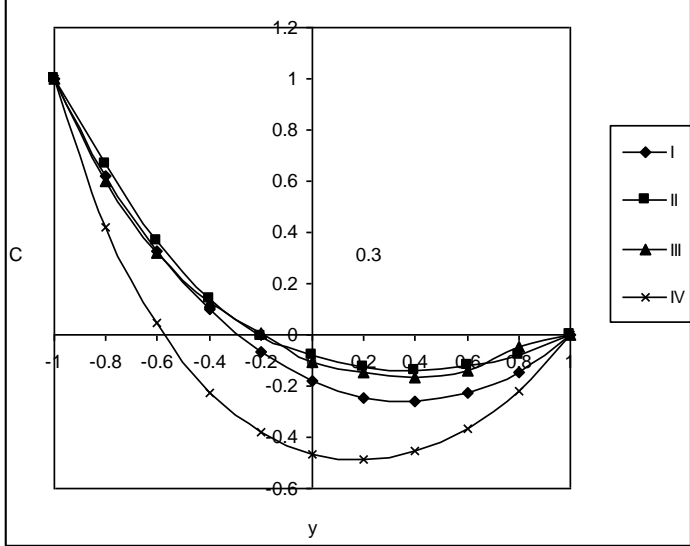

Fig. 20 : Variation of $\mathrm{C}$ with $\mathrm{k}$

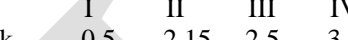

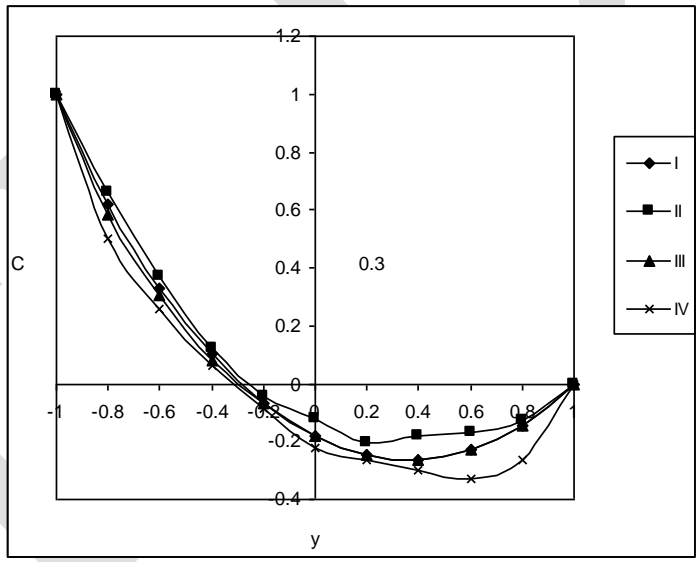

Fig. 22 : Variation of $C$ with $x+\gamma t$

$\begin{array}{rrrr}\text { I } & \text { II } & \text { III } & \text { IV } \\ \mathrm{x}+\gamma \mathrm{t} & \pi / 4 & \pi / 2 & \pi\end{array}$

Table - 1

Average Nusselt number $(\mathrm{Nu})$ at $\mathrm{y}=+1$

$\begin{array}{cccccccccc}\text { G } & \text { I } & \text { II } & \text { III } & \text { IV } & \text { V } & \text { VI } & \text { VII } & \text { VIII } & \text { IX } \\ 5 & -3.6518 & -3.7847 & -3.1927 & -0.1312 & -0.2451 & -0.3523 & -4.8450 & -3.3007 & -3.4880 \\ 10 & -6.9276 & -7.0884 & -6.922 & 1.084 & 2.2547 & 3.8702 & -11.5524 & -5.4567 & -6.5127 \\ -5 & -2.897 & -2.9905 & -2.6277 & -0.6522 & -0.7946 & -0.8967 & -3.7351 & -2.7858 & -2.9063 \\ -10 & -3.6722 & -3.9906 & -3.4430 & -0.3846 & 0.4917 & 0.7963 & -6.6323 & -3.1106 & -3.44 \\ \alpha & 2 & 4 & 6 & -2 & -4 & -6 & 2 & 2 & 2 \\ \mathrm{~N} & 1 & 1 & 1 & 1 & 1 & 1 & 2 & -0.5 & -0.8\end{array}$

Table - 2

Average Nusselt number ( $\mathrm{Nu}$ ) at $\mathrm{y}=+1$

$\begin{array}{ccccccccccc}\mathbf{G} & \mathbf{I} & \mathbf{I I} & \mathbf{I I I} & \mathbf{I V} & \mathbf{V} & \mathbf{V I} & \mathbf{V I I} & \mathbf{V I I I} & \mathbf{I X} & \mathbf{X} \\ 5 & -5.2969 & -3.7951 & -3.6518 & -3.6441 & -4.4502 & -3.5157 & -4.4453 & -2.9705 & -7.5890 & -5.6034 \\ 10 & -26.1043 & -8.1426 & -6.9276 & -6.4599 & -9.9468 & -6.8410 & -12.24 & -5.3542 & -18.5823 & -12.4468 \\ -5 & -1.9620 & -2.6693 & -2.897 & -2.4333 & -3.5480 & -2.7663 & -3.1582 & -2.5361 & -4.7331 & -3.7909 \\ -10 & -2.0446 & -3.1310 & -3.6722 & -2.113 & -5.9795 & -3.1125 & -4.1570 & -3.3175 & -5.5041 & -34.6564 \\ \mathrm{Sc} & 0.24 & 0.6 & 1.3 & 2.01 & 1.3 & 1.3 & 1.3 & 1.3 & 1.3 & 1.3 \\ \mathrm{~S}_{0} & 0.5 & 0.5 & 0.5 & 0.5 & 1 & -0.5 & 1 & 0.5 & 0.5 & 0.5 \\ \mathrm{x}+\gamma \mathrm{t} & \pi / 4 & \pi / 4 & \pi / 4 & \pi / 4 & \pi / 4 & \pi / 4 & \pi / 4 & \pi / 2 & \pi & 2 \pi\end{array}$




\section{Table -3}

$\begin{array}{cccccccccc}\mathbf{G} & \mathbf{I} & \mathbf{I I} & \mathbf{I I I} & \mathbf{I V} & \mathbf{V} & \mathbf{V I} & \mathbf{V I I} & \text { VIII } & \text { IX } \\ 5 & -3.6518 & -3.3782 & -3.6044 & -7.8117 & -4.1874 & -4.4743 & -4.6530 & -4.7758 & \\ 10 & -6.9276 & -5.0249 & -5.7493 & -21.3136 & -15.8843 & -27.8575 & -44.6801 & -70.0461 & \\ -5 & -2.8970 & -2.9046 & -3.1165 & -5.7238 & -2.9433 & -2.9850 & -3.1158 & -3.2602 & \\ -10 & -3.6722 & -3.4805 & -4.1126 & -11.7103 & -3.9858 & -4.0828 & -4.1300 & -4.1580 & \\ \mathrm{k} & 0.5 & 1.5 & 2.5 & 3.5 & 0.5 & 0.5 & 0.5 & 0.5 & \\ \mathrm{Ec} & 0.01 & 0.01 & 0.01 & 0.01 & 0.03 & 0.05 & 0.07 & 0.09 & \end{array}$

Table -4

Average Nusselt number ( $\mathrm{Nu}$ ) at $\mathrm{y}=\mathbf{- 1}$

$\begin{array}{cccccccccc}\mathbf{G} & \mathbf{I} & \text { II } & \text { III } & \text { IV } & \text { V } & \text { VI } & \text { VII } & \text { VIII } & \text { IX } \\ 5 & 0.3243 & 0.2262 & 0.2032 & 0.1288 & 0.1517 & 0.1626 & 0.2861 & 0.3653 & 0.3641 \\ 10 & 0.5450 & 0.3015 & 0.2574 & 0.1492 & 0.1852 & 0.2047 & 0.3486 & 0.7235 & 0.6840 \\ -5 & 0.1801 & 0.1512 & 0.1448 & 0.1022 & 0.1112 & 0.1153 & 0.1868 & 0.1635 & 0.1595 \\ -10 & 0.1476 & 0.1305 & 0.1272 & 0.9301 & 0.9930 & 1.0006 & 0.1518 & 0.1234 & 0.1175 \\ \alpha & 2 & 4 & 6 & -2 & -4 & -6 & 2 & 2 & 2 \\ \mathrm{~N} & 1 & 1 & 1 & 1 & 1 & 1 & 2 & -0.5 & -0.8\end{array}$

Table -5

$\begin{array}{ccccccccccc}\mathbf{G} & \mathbf{I} & \mathbf{I I} & \mathbf{I I I} & \mathbf{I V} & \mathbf{V} & \mathbf{V I} & \mathbf{V I I} & \mathbf{V I I I} & \mathbf{I X} & \mathbf{X} \\ 5 & 0.6647 & 0.377 & 0.3243 & 0.2973 & 0.2821 & 0.4093 & 0.4412 & 0.1932 & -0.2162 & -0.8504 \\ 10 & -0.1019 & 0.8877 & 0.5450 & 0.4006 & 0.3452 & 1.0178 & 1.0051 & 0.2465 & 0.2584 & -0.3733 \\ -5 & 0.1659 & 0.1749 & 0.1801 & 0.203 & 0.1912 & 0.1545 & 0.1416 & 0.1354 & -0.7442 & 0.8608 \\ -10 & 0.1221 & 0.1263 & 0.1476 & 0.1779 & 0.1596 & 0.1127 & 0.9582 & 0.1179 & 0.3766 & 0.4581 \\ \mathrm{Sc} & 0.24 & 0.6 & 1.3 & 2.01 & 1.3 & 1.3 & 1.3 & 1.3 & 1.3 & 1.3 \\ \mathrm{~S}_{0} & 0.5 & 0.5 & 0.5 & 0.5 & 1 & -0.5 & 1 & 0.5 & 0.5 & 0.5 \\ \mathrm{x}+\gamma \mathrm{t} & \pi / 4 & \pi / 4 & \pi / 4 & \pi / 4 & \pi / 4 & \pi / 4 & \pi / 4 & \pi / 2 & \pi & 2 \pi\end{array}$

Table -6

\begin{tabular}{ccccccccc}
\multicolumn{8}{c}{ Average Nusselt number $(\mathbf{N u})$ at $\mathbf{y}=\mathbf{- 1}$} \\
$\mathbf{G}$ & $\mathbf{I}$ & II & III & IV & $\mathbf{V}$ & VI & VII & VIII \\
5 & 0.3243 & 0.3329 & 0.3663 & 0.4256 & 0.1976 & 0.1427 & 0.1121 & 0.0958 \\
10 & 0.5450 & 0.4492 & 0.4622 & 0.5131 & 0.7038 & 0.6004 & 0.5781 & 0.4672 \\
-5 & 0.1801 & 0.2284 & 0.2669 & 0.2952 & 0.3439 & 0.2684 & 0.2076 & 0.1612 \\
-10 & 0.1476 & 0.2011 & 0.2384 & 0.2646 & 0.6656 & 0.4485 & 0.3478 & 0.2897 \\
$\mathrm{k}$ & 0.5 & 1.5 & 2.5 & 3.5 & 0.5 & 0.5 & 0.5 & 0.5 \\
$\mathrm{Ec}$ & 0.01 & 0.01 & 0.01 & 0.01 & 0.03 & 0.05 & 0.07 & 0.09
\end{tabular}

Table -7

Average Sherwood number (Sh) at $y=+1$

$\begin{array}{cccccccccc}\text { G } & \text { I } & \text { II } & \text { III } & \text { IV } & \text { V } & \text { VI } & \text { VII } & \text { VIII } & \text { IX } \\ 5 & -0.5287 & -0.6748 & -0.7370 & 1.2742 & -6.5505 & -2.4893 & -0.5791 & -0.4578 & -0.4461 \\ 10 & -0.4333 & -0.6965 & 0.8224 & 2.4508 & -3.4772 & -1.8945 & -0.5741 & -0.1991 & -0.1720 \\ -5 & -0.3935 & -0.4444 & -0.5728 & 0.6914 & 4.0921 & -2.0803 & -0.4276 & -0.4609 & -0.4767 \\ -10 & -0.0733 & 1.21 & 2.6298 & 1.3617 & -6.4916 & -2.959 & -0.1131 & -0.2650 & -0.2193 \\ \alpha & 2 & 4 & 6 & -2 & -4 & -6 & 2 & 2 & 2 \\ \mathbf{N} & 1 & 1 & 1 & 1 & 1 & 1 & 2 & -0.5 & -0.8\end{array}$




\section{Table -8}

\begin{tabular}{ccccccccccc} 
& \multicolumn{10}{c}{ Average Sherwood number (Sh) at $\mathbf{y}=+\mathbf{1}$} \\
$\mathbf{G}$ & $\mathbf{I}$ & II & III & $\mathbf{I V}$ & $\mathbf{V}$ & $\mathbf{V I}$ & $\mathbf{V I I}$ & VIII & IX & $\mathbf{X}$ \\
5 & -0.4598 & -0.4628 & -0.5287 & -0.5943 & -0.7565 & 0.2249 & 0.6823 & -0.5680 & -0.6082 & -0.5082 \\
10 & -0.5826 & -0.6788 & -0.8333 & -0.9178 & -0.9038 & 0.1758 & -0.2186 & -0.4639 & -0.5332 & -0.4036 \\
-5 & -0.1196 & -0.2436 & -0.3935 & -0.5078 & -0.4951 & 0.4018 & -0.5945 & -0.4235 & -0.4632 & -0.3236 \\
-10 & 0.1239 & 0.1471 & -0.1733 & -0.2453 & -0.2051 & -0.0596 & -0.1665 & -0.1030 & -0.1234 & -0.0486 \\
$\mathrm{Sc}$ & 0.24 & 0.6 & 1.3 & 2.01 & 1.3 & 1.3 & 1.3 & 1.3 & 1.3 & 1.3 \\
$\mathrm{~S}_{0}$ & 0.5 & 0.5 & 0.5 & 0.5 & 1 & -0.5 & 1 & 0.5 & 0.5 & 0.5 \\
$\mathrm{x}+\gamma \mathrm{t}$ & $\pi / 4$ & $\pi / 4$ & $\pi / 4$ & $\pi / 4$ & $\pi / 4$ & $\pi / 4$ & $\pi / 4$ & $\pi / 2$ & $\pi$ & $2 \pi$
\end{tabular}

Table - 9

$\begin{array}{ccccccccc}\mathbf{G} & \mathbf{I} & \mathbf{I I} & \mathbf{I I I} & \mathbf{I V} & \mathbf{V} & \mathbf{V I} & \mathbf{V I I} & \text { VIII } \\ 5 & -0.5287 & -0.4116 & -0.368 & -0.9962 & -0.4938 & -0.4619 & -0.4327 & -0.4059 \\ 10 & -0.4333 & -0.2248 & -0.1874 & -1.1292 & -0.2808 & 0.1929 & 0.1606 & 0.1257 \\ -5 & -0.3935 & -0.3916 & -0.3440 & 0.3623 & 0.2408 & 0.1923 & 0.1492 & -0.12757 \\ -10 & -0.0733 & -0.1686 & -0.1406 & 1.06 & 0.1269 & -0.0967 & -0.0655 & -0.0238 \\ \mathrm{k} & 0.5 & 1.5 & 2.5 & 3.5 & 0.5 & 0.5 & 0.5 & 0.5 \\ \mathrm{Ec} & 0.01 & 0.01 & 0.01 & 0.01 & 0.03 & 0.05 & 0.07 & 0.09\end{array}$

Table -10

Average Sherwood number (Sh) at $y=-1$

$\begin{array}{cccccccccc}\mathbf{G} & \text { I } & \text { II } & \text { III } & \text { IV } & \text { V } & \text { VI } & \text { VII } & \text { VIII } & \text { IX } \\ 5 & -1.3466 & -1.8557 & -2.6933 & 0.7148 & -0.214 & -0.1212 & -0.9263 & -1.9460 & -2.0351 \\ 10 & -1.6755 & -6.1348 & 1.3695 & 1.2552 & 0.7032 & 0.6537 & -0.5717 & -4.3179 & -4.5899 \\ -5 & -2.0065 & -2.7624 & -3.7138 & 1.4081 & -1.3852 & -0.8868 & -2.1060 & -2.1265 & -2.1467 \\ -10 & -3.8765 & -5.3172 & 6.2184 & 2.6404 & 0.5818 & 0.4898 & -3.9760 & -4.0760 & -4.1769 \\ \alpha & 2 & 4 & 6 & -2 & -4 & -6 & 2 & 2 & 2 \\ \mathrm{~N} & 1 & 1 & 1 & 1 & 1 & 1 & 2 & -0.5 & -0.8\end{array}$

Table - 11

\begin{tabular}{ccccccccccc}
\multicolumn{10}{c}{} & \multicolumn{10}{c}{ Average Sherwood number (Sh) at $\mathbf{y}=\mathbf{- 1}$} \\
$\mathbf{G}$ & $\mathbf{I}$ & II & III & $\mathbf{I V}$ & $\mathbf{V}$ & $\mathbf{V I}$ & $\mathbf{V I I}$ & $\mathbf{V I I I}$ & $\mathbf{I X}$ & $\mathbf{X}$ \\
5 & -0.5231 & -0.9076 & -1.3466 & -1.6495 & -0.9892 & 1.4520 & 2.5458 & -1.3666 & -1.4460 & -1.3066 \\
10 & -0.2608 & -0.8967 & -1.6755 & 2.2551 & -0.6648 & 1.3346 & 3.1633 & -1.7750 & -1.8765 & -1.6055 \\
-5 & -1.4003 & -1.6489 & -2.0065 & -2.2422 & -2.6256 & 3.9487 & -1.4056 & -2.1062 & -2.2060 & -1.9665 \\
-10 & -2.1889 & -2.8352 & -3.8765 & -4.5181 & -4.7329 & 2.9441 & -1.2885 & -3.9769 & -4.0765 & 0.8007 \\
$\mathrm{Sc}$ & 0.24 & 0.6 & 1.3 & 2.01 & 1.3 & 1.3 & 1.3 & 1.3 & 1.3 & 1.3 \\
$\mathrm{~S}_{0}$ & 0.5 & 0.5 & 0.5 & 0.5 & 1 & -0.5 & 1 & 0.5 & 0.5 & 0.5 \\
$\mathrm{x}+\gamma \mathrm{t}$ & $\pi / 4$ & $\pi / 4$ & $\pi / 4$ & $\pi / 4$ & $\pi / 4$ & $\pi / 4$ & $\pi / 4$ & $\pi / 2$ & $\pi$ & $2 \pi$
\end{tabular}

Table - 12

Average Sherwood number (Sh) at $y=-1$

$\begin{array}{ccccccccc}\text { G } & \text { I } & \text { II } & \text { III } & \text { IV } & \text { V } & \text { VI } & \text { VII } & \text { VIII } \\ 5 & -1.3466 & -2.3778 & -3.4375 & -3.6104 & -1.4475 & -2.1621 & -3.0878 & -4.0225 \\ 10 & -1.6755 & -3.7239 & -5.4016 & -0.6766 & -2.1955 & -2.7964 & -3.4986 & -4.3303 \\ -5 & -2.0065 & -2.5340 & -3.3429 & -4.2554 & -5.0494 & -6.6016 & 8.6715 & 11.102 \\ -10 & -3.8765 & -4.3399 & -4.9446 & -3.3268 & 8.7239 & 11.7719 & 12.8861 & 13.5159 \\ \mathrm{k} & 0.5 & 1.5 & 2.5 & 3.5 & 0.5 & 0.5 & 0.5 & 0.5 \\ \mathrm{Ec} & 0.01 & 0.01 & 0.01 & 0.01 & 0.03 & 0.05 & 0.07 & 0.09\end{array}$




\section{REFERENCES}

1. Guria,M and Jana,R.N:Hydrodynamic flows through vertical wavy channel with traveling thermal waves embedded in porous medium,Int.J.Appl.Math and Mech,V.11,N0.3,pp.609-621(2006)

2. Nagaraja, $\mathbf{P}:$ Heat and Mass Transportation in time dependent flows through channels, Ph.D. thesis S.K.University, Anantapur, India (1973).

3. Puroshotama reddy, Y :Unsteady mixed convection flows through vertical channel with varying gap. Ph.D thesis S.K.Univeristy, Anantapur, India (1995).

4. Ravindra, M :MHD convection flow through a porous medium with non-uniform wall temperature, Ph.D thesis, S.K.Uiversity, Anantapur, India (1994).

5. Sivanjaneya Prasad, $P$ :Effects of convecive heat and mass transfer on unsteady hydromagnetic channel flows, Ph.D, thesis, S.K.University, Anantapur , India(2002).

6. Sulochana et al : Connective Heat and Mass Transfer through a porous medium in channels -A finite Element approach, Ph .D thesis submitted to S.K.University, Anatapur.

7. Tanmay Basak, S.Roy,T.Paul,I.Pop, Natural convection in a square cavity filled with a porous medium. Effects of various thermal boundary conditions. Intl. J.of Heat and mass transfer 49 (2006) 1430-1441.

8. Vajravelu, K and Debnath, L : Non -Linear study of convective heat transfer and fluid flows induced by traveling thermal waves ,Acta Mech, V.59, PP-233-249 (1986).

9. White head, J.A : Geo Physics fluid dynamics V.3, PP.161-180 (1972). 